\title{
Stability conditions for linear continuous-time fractional-order state-delayed systems
}

\author{
M. BUSŁOWICZ * \\ Faculty of Electrical Engineering, Bialystok University of Technology, 45D Wiejska St., 15-351 Bialystok, Poland
}

\begin{abstract}
The stability problem of continuous-time linear fractional order systems with state delay is considered. New simple necessary and sufficient conditions for the asymptotic stability are established. The conditions are given in terms of eigenvalues of the state matrix and time delay. It is shown that in the complex plane there exists such a region that location in this region of all eigenvalues of the state matrix multiplied by delay in power equal to the fractional order is necessary and sufficient for the asymptotic stability. Parametric description of boundary of this region is derived and simple new analytic necessary and sufficient conditions for the stability are given. Moreover, it is shown that the stability of the fractional order system without delay is necessary for the stability of this system with delay. The considerations are illustrated by a numerical example.
\end{abstract}

Key words: linear system, fractional, continuous-time, state delay, asymptotic stability.

\section{Introduction}

Dynamical systems described by fractional order differential or difference equations have been investigated in several areas such as viscoelasticity, electrochemistry, diffusion processes, automatic control, power electronic, etc. The problem of analysis and synthesis of dynamical systems described by fractional order differential (or difference) equations has been considered in monographs [1-4], for example.

The problems of stability of linear continuous-time and discrete-time fractional order systems, standard and positive, have been investigated in the above mentioned monographs and in many papers, see [5-12] for example, and references therein.

In last years the stability problem of fractional systems with delays has been considered in [13-19].

The aim of the paper is to give the methods (graphical and analytic) for asymptotic stability checking for fractional order continuous-time linear systems with state delay. The stability problem of such systems has not been considered yet. The stability problem of standard (i.e. non-fractional) continuoustime linear systems with pure delay has been considered in [20-24].

In the paper the following notations is used: $\Re^{n \times m}-$ the set of $n \times m$ real matrices and $\Re^{n}=\Re^{n \times 1} ; I$ - the identity matrix.

\section{Preliminaries and problem formulation}

Consider a continuous-time linear system of fractional order with pure state delay described by the state equation

$$
D_{t}^{\alpha} x(t)=A x(t-h)+B u(t), \quad 0<\alpha<2,
$$

where $x(t) \in \Re^{n}, u(t) \in \Re^{m}, A \in \Re^{n \times n}, B \in \Re^{n \times m}, h \in \Re$ is a delay and

$$
D_{t}^{\alpha} x(t)=\frac{1}{\Gamma(p-\alpha)} \int_{0}^{t} \frac{x^{(p)}(\tau) d \tau}{(t-\tau)^{\alpha+1-p}}, \quad p-1 \leq \alpha \leq p,
$$

is the Caputo definition of the fractional $\alpha$-order derivative, where $x^{(p)}(t)=d^{p} x(t) / d t^{p}$ ( $p$ is a natural number) and $\Gamma(\alpha)$ is the Euler gamma function.

The characteristic function (quasi-polynomial in $s^{\alpha}$ and $\left.e^{-s h}\right)$ of the system (1) can be computed from the formula

$$
\begin{gathered}
q(s)=\operatorname{det}\left(I s^{\alpha}-A e^{-s h}\right)=\prod_{i=1}^{n} q_{i}(s) \\
=\prod_{i=1}^{n}\left(s^{\alpha}-\lambda_{i} e^{-s h}\right),
\end{gathered}
$$

where

$$
q_{i}(s)=s^{\alpha}-\lambda_{i} e^{-s h}
$$

and $\lambda_{i}=u_{i}+j v_{i}$ is the $i$-th eigenvalue of the matrix $A$ $(i=1, \ldots, n)$.

The fractional system (1) is bounded-input boundedoutput (BIBO) stable (shortly stable) if and only if $q(s)$ has no poles with non-negative real parts, i.e.

$$
q(s)=\prod_{i=1}^{n}\left(s^{\alpha}-\lambda_{i} e^{-s h}\right) \neq 0 \quad \text { for } \quad \operatorname{Re} s>0 .
$$

The condition (5) can be written in the form of $n$ conditions

$$
q_{i}(s) \neq 0 \quad \text { for } \operatorname{Re} s>0, \quad i=1, \ldots, n .
$$

The characteristic quasi-polynomial which has no poles with non-negative real parts is called the stable quasipolynomial.

*e-mail: andrusz@pb.bialystok.pl 
The aim of the paper is to give the methods for checking the conditions (6) and (5).

\section{The main result}

First of all we consider the stability problem for the fractional quasi-polynomial (4), where $\lambda_{i}=u_{i}+j v_{i}$ is a complex number.

A root location of (4) in the open left-half plane is equivalent to the root location in this plane of the quasi-polynomial

$$
w_{i}(z)=z^{\alpha} e^{z}-\widetilde{\lambda}_{i}
$$

where $\widetilde{\lambda}_{i}=\widetilde{u}_{i}+j \widetilde{v}_{i}, \widetilde{u}_{i}=h^{\alpha} u_{i}, \widetilde{v}_{i}=h^{\alpha} v_{i}$.

We apply the $D$ decomposition method of Nejmark (see [23] for the state of the art and [10] as an example of application) for determining the stability region of (4) in the complex $\left(\widetilde{u}_{i}, \widetilde{v}_{i}\right)$-plane.

Substituting $z=j \omega$ in (7) and equating to zero we obtain the parametric description of boundary of the stability region in the complex $\left(\widetilde{u}_{i}, \widetilde{v}_{i}\right)$-plane. This boundary is a part of the curve with the parametric description

$$
(j \omega)^{\alpha} e^{j \omega}=\widetilde{u}_{i}(\omega)+j \widetilde{v}_{i}(\omega)
$$

for $\omega \in(-\infty, \infty)$.

Taking account that $(j \omega)^{\alpha}=\omega^{\alpha} e^{j \alpha \pi / 2}$, Eq. (8) for $\omega>0$ can be written in the form of two real equations

$$
\begin{aligned}
& \widetilde{u}_{i}(\omega)=\omega^{\alpha} \cos (\omega+\alpha \pi / 2), \\
& \widetilde{v}_{i}(\omega)=\omega^{\alpha} \sin (\omega+\alpha \pi / 2) .
\end{aligned}
$$

Since $(-j \omega)^{\alpha}=\omega^{\alpha} e^{-j \alpha \pi / 2}(\omega>0)$ one has

$$
(-j \omega)^{\alpha} e^{-j \omega}=\widetilde{u}_{i}(\omega)-j \widetilde{v}_{i}(\omega), \quad \omega \in(-\infty, \infty),
$$

where $\widetilde{u}_{i}(\omega)$ and $\widetilde{v}_{i}(\omega)$ are defined by (9).

This means that the curve described by (8) (or (9), equivalently) for $\omega \in(-\infty, \infty)$ is symmetric with respect to the real axis of the complex $\left(\widetilde{u}_{i}, \widetilde{v}_{i}\right)$-plane.

From (9) it follows that $\widetilde{v}_{i}(\omega)=0$ for $\omega= \pm \omega_{b}$ where $w_{b}=\pi-\alpha \pi / 2$ and

$$
\widetilde{u}_{i}\left(\omega_{b}\right)=\widetilde{u}_{i}\left(-\omega_{b}\right)=-\omega_{b}^{\alpha}=-[\pi(1-\alpha / 2)]^{\alpha} .
$$

Hence, the curve (8) (or (9)) for $\omega \in\left[-\omega_{b}, \omega_{b}\right]$ is a closed curve. This curve divides the complex $\left(\widetilde{u}_{i}, \widetilde{v}_{i}\right)$-plane into two regions, one bounded and one unbounded (see Fig. 1). Denote by $S(\alpha)$ the bounded region.

The quasi-polynomial (7) for $z=j \omega$ can be written in the form $w_{i}(j \omega)=P_{i}(\omega)+j Q_{i}(\omega)$, where $P_{i}(\omega)=\omega^{\alpha} \cos (\omega+$ $\alpha \pi / 2)-\widetilde{u}_{i}(\omega), Q_{i}(\omega)=\omega^{\alpha} \sin (\omega+\alpha \pi / 2)-\widetilde{v}_{i}(\omega)$. If $\omega$ increases in the interval $\left[-\omega_{b}, \omega_{b}\right]$ from $-\omega_{b}$ to $\omega_{b}$ then the point $w_{i}(j \omega)$ moves along the boundary (8) in the positive direction. From this and positivity of the Jacobian

$$
J(\omega)=\operatorname{det}\left[\begin{array}{ll}
\frac{\partial P_{i}(\omega)}{\partial \widetilde{u}_{i}(\omega)} & \frac{\partial P_{i}(\omega)}{\partial \widetilde{v}_{i}(\omega)} \\
\frac{\partial Q_{i}(\omega)}{\partial \widetilde{u}_{i}(\omega)} & \frac{\partial Q_{i}(\omega)}{\partial \widetilde{v}_{i}(\omega)}
\end{array}\right]=1
$$

it follows that the stability region lies on the left of the boundary (8) [24]. This means that $S(\alpha)$ is the stability region of the quasi-polynomial (7) and also (4).

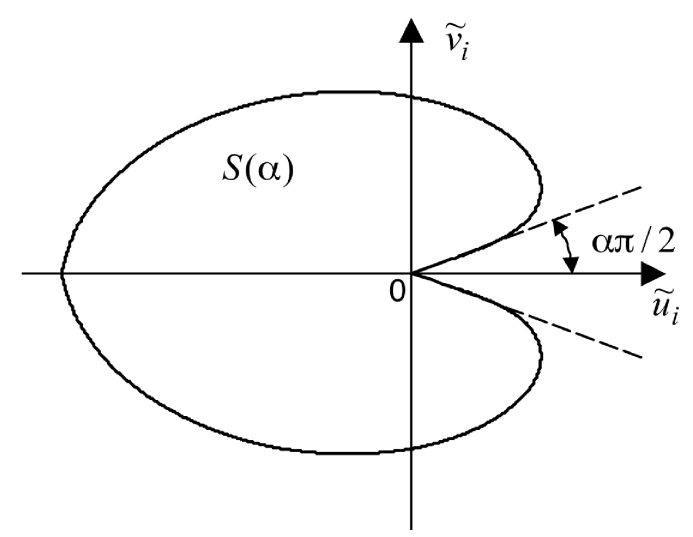

Fig. 1. Stability region $S(\alpha)$ for $0<\alpha<1$

From the above we have the following lemma.

Lemma 1. The quasi-polynomial (7) (and (4)) is stable if and only if the complex number $\lambda_{i}=u_{i}+j v_{i}$ multiplied by $h^{\alpha}$ (i.e. $\widetilde{\lambda}_{i}=\widetilde{u}_{i}+j \widetilde{v}_{i}$ with $\widetilde{u}_{i}=h^{\alpha} u_{i}$, $\widetilde{v}_{i}=h^{\alpha} v_{i}$ ) lies in the complex $\left(\widetilde{u}_{i}, \widetilde{v}_{i}\right)$-plane in the stability region $S(\alpha)$ with the boundary (8) for $\omega \in\left[-\omega_{b}, \omega_{b}\right]$.

From (9) for $\omega=0$ we have $\widetilde{u}_{i}(0)=\widetilde{v}_{i}(0)=0$ and

$$
\tan \left|\frac{\widetilde{v}_{i}(0)}{\widetilde{u}_{i}(0)}\right|=\tan \frac{\alpha \pi}{2} .
$$

This means that for any point $\widetilde{\lambda}_{i}=\widetilde{u}_{i}+j \widetilde{v}_{i}$ in the stability region $S(\alpha)$ the following condition holds

$$
\left|\arg \widetilde{\lambda}_{i}\right|>\alpha \pi / 2 \text {, }
$$

where $\arg \widetilde{\lambda}_{i} \in(-\pi, \pi]$ denotes the main argument of the complex number $\widetilde{\lambda}_{i}$.

The main argument of $\lambda_{i}=u_{i}+j v_{i}$ can be computed from the formula

$$
\arg \lambda_{i}=\operatorname{sgn}\left(v_{i}\right) \cdot \arccos \left(u_{i} /\left|\lambda_{i}\right|\right) .
$$

The stability regions $S(\alpha)$ in the complex $\left(\widetilde{u}_{i}, \widetilde{v}_{i}\right)$-plane are shown in Figs. 1 and 2 for $0<\alpha<1$ and for $1<\alpha<2$, respectively. Figure 3 shows the stability regions $S(\alpha)$ for a few values of fractional order $0<\alpha<2$. For $\alpha \geq 2$ the stability regions $S(\alpha)$ are empty sets.

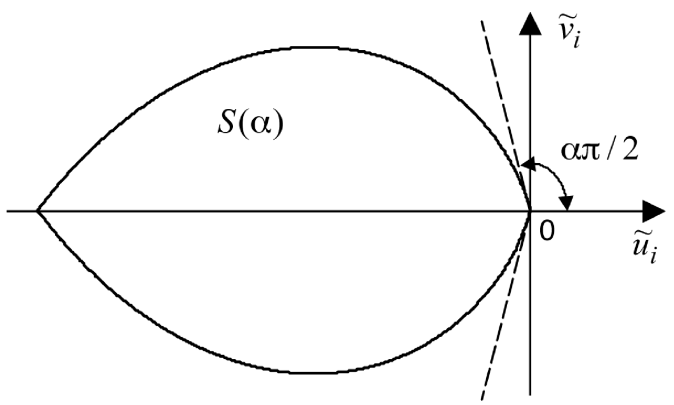

Fig. 2. Stability region $S(\alpha)$ for $1<\alpha<2$ 


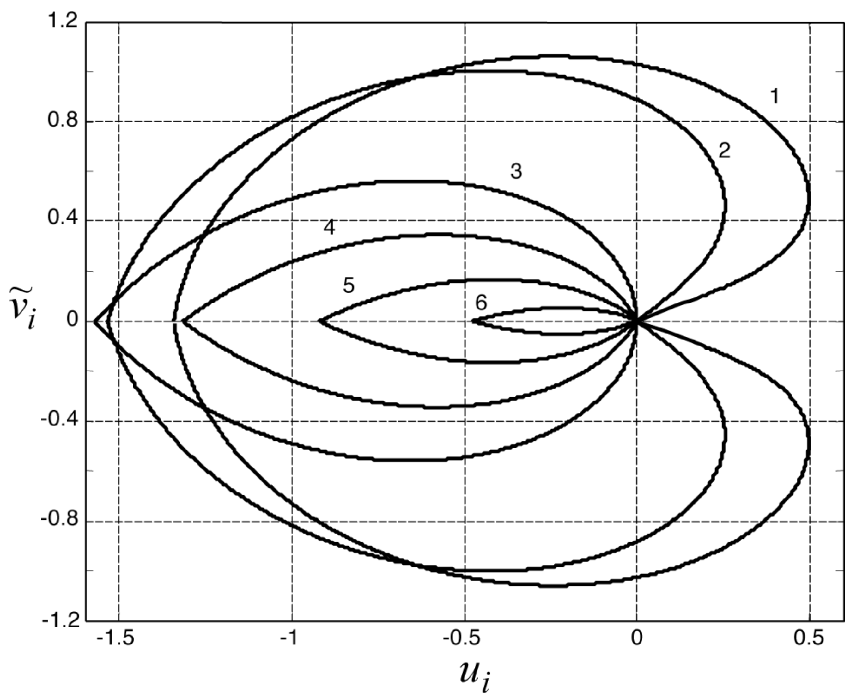

Fig. 3. Stability regions $S(\alpha)$ for a few values of $\alpha$ : $\alpha=0.3$ (boundary 1 ); $\alpha=0.5$ (boundary 2); $\alpha=1$ (boundary 3 ); $\alpha=1.2$ (boundary 4 ); $\alpha=1.4$ (boundary 5 ); $\alpha=1.6$ (boundary 6 )

For $\alpha=1$ the stability region $S(\alpha)$ is the asymptotic stability region of the natural order system with pure state delay $\dot{x}(t)=A x(t-h)$ [20]. This region is shown in Fig. 3 as the region with boundary denoted by number 3 .

From (3) and the above consideration we have the following theorem and remarks.

Theorem 1. The fractional system (1) with pure state delay is stable if and only if all eigenvalues $\lambda_{i}, i=1, \ldots, n$, of the matrix $A$ multiplied by $h^{\alpha}$ (i.e. $\widetilde{\lambda}_{i}=\widetilde{u}_{i}+j \widetilde{v}_{i}$ ) lie in the open region $S(\alpha)$ in the complex $\left(\widetilde{u}_{i}, \widetilde{v}_{i}\right)$-plane with the boundary (8) (or (9)) for $\omega \in\left[-\omega_{b}, \omega_{b}\right]$.

Recall that satisfaction of (13) for $i=1, \ldots, n$, is the necessary and sufficient condition for stability of the system (1) without delay, that is of the system $D_{t}^{\alpha} x(t)=A x(t)+B u(t)$, $0<\alpha<2$ (here $\tilde{\lambda}_{i}=\lambda_{i}=u_{i}+j v_{i}$ is the $i$-th eigenvalue of $A$ ).

Remark 1. Stability of the fractional system (1) without delay is necessary for stability of this system with delay.

Remark 2. Asymptotic stability of the matrix $A$ (all eigenvalues have negative real parts) is necessary for stability of the system (1) for $1<\alpha<2$. For $0<\alpha<1$ eigenvalues of $A$ may have positive real parts. Moreover, the system for $0<\alpha<1$ may be stable when all eigenvalues of $A$ are complex conjugate with positive real parts.

Remark 3. The fractional system (1) is unstable if the matrix $A$ has at least one non-negative real eigenvalue. In particular, this holds if $\operatorname{det} A=0$.

Remark 4. If the fractional system (1) is stable then real eigenvalues of $A$ are negative and greater than $-[\pi(1-$ $\alpha / 2)]^{\alpha} / h^{\alpha}$.

The condition of Theorem 1 can be written in the analytic form as follows.
Theorem 2. The fractional system (1) with pure state delay is stable if and only if for all eigenvalues $\lambda_{i}=u_{i}+j v_{i}$, $i=1,2, \ldots, n$, of the matrix $A$ the following two conditions hold

$$
\begin{aligned}
& \left|\arg \lambda_{i}\right|>\alpha \pi / 2, \\
& h^{\alpha}\left|\lambda_{i}\right|<\left|\omega_{0 i}\right|^{\alpha},
\end{aligned}
$$

where $\arg \lambda_{i} \in(-\pi, \pi]$ denotes the main argument of the eigenvalue $\lambda_{i}$ and

$$
\omega_{0 i}=\arg \lambda_{i}-\operatorname{sgn}\left(v_{i}\right) \cdot \alpha \pi / 2 .
$$

Proof. Since $\varphi_{i}=\arg \lambda_{i}=\arg \lambda_{i} h^{\alpha}=\arg \tilde{\lambda}_{i}$ (see Fig. 4), satisfaction of (15) for $i=1,2, \ldots, n$ is necessary for the stability of the fractional system (1).

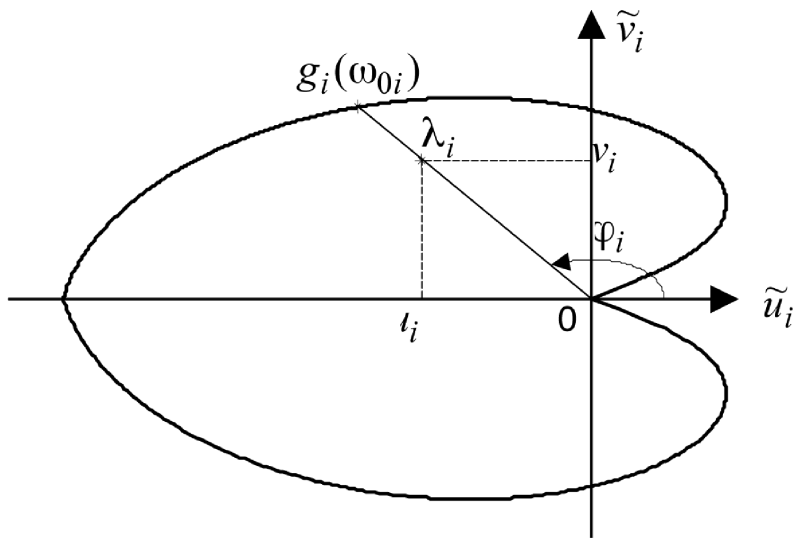

Fig. 4. Plot for the proof of Theorem 2

From Fig. 4 it follows that the point $\widetilde{\lambda}_{i}=\widetilde{u}_{i}+j \widetilde{v}_{i}=$ $h^{\alpha}\left|\lambda_{i}\right| e^{j \varphi_{i}}$ lies in $S(\alpha)$ if and only if $h^{\alpha}\left|\lambda_{i}\right|<\left|g_{i}\left(\omega_{0 i}\right)\right|$, where $\omega_{0 i}$ is defined by (17) and $g\left(\omega_{0 i}\right)=\left(j \omega_{0 i}\right)^{\alpha} e^{j \omega_{0 i}}$. Because $\left|g\left(\omega_{0 i}\right)\right|=\left|\left(j \omega_{0 i}\right)^{\alpha} e^{j \omega_{0 i}}\right|=\left|\omega_{0 i}\right|^{\alpha}$ the condition (16) must be satisfied. This completes the proof.

Remark 5. It is interesting to note that the form of the stability criterion (15), (16) is quite similar to the stability criterion of Ref. [9], in that it includes both the phase/argument condition of Matignon [5] and, additionally, the modulus condition.

From Theorem 2 we have the following corollary.

Corollary 1. If the fractional system (1) with pure state delay is stable for $h=0$ then it is stable for all $h \in\left[0, h_{0}\right)$, $h_{0}=\min _{i}\left\{h_{i}\right\}$, where

$$
h_{i}=\exp \left(\frac{\ln \left(\left|\omega_{0 i}\right|^{\alpha} /\left|\lambda_{i}\right|\right)}{\alpha}\right), \quad i=1,2, \ldots, n .
$$

Proof. From (16) it follows that $h_{i}^{\alpha}=\left|\omega_{0 i}\right|^{\alpha} /\left|\lambda_{i}\right|$. Computing $h_{i}$ from this equality one obtains (18).

Remark 6. If $\lambda_{i}=u_{i}$ is real and negative then $\left|\lambda_{i}\right|=\left|u_{i}\right|$ and $\left|\omega_{0 i}\right|=[\pi(1-\alpha / 2)]$.

Remark 7. It is sufficient to check the stability conditions given in Theorems 1 and 2 only for real and complex eigenvalues of $A$ with positive imaginary parts. This follows from the fact that complex eigenvalues of $A$ are pair-wise conjugate and the stability region $S(\alpha)$ is symmetric with respect to real axis. 
If $\alpha=1$ then the stability region $S(\alpha)$ has the form shown in Fig. 3 (boundary 3). From (15) and (16) we obtain, respectively, $\left|\arg \lambda_{i}\right|>\pi / 2$, which is equivalent to $u_{i}<0$, and $h\left|\lambda_{i}\right|<\left|\omega_{0 i}\right|$. From (14) for $v_{i} \geq 0$ it follows that $\arg \lambda_{i}=\arctan \left(\left|v_{i}\right| /\left|u_{i}\right|\right)$ and by (17) $\left|\omega_{0 i}\right|=$ $\left|\arctan \left(\left|v_{i}\right| /\left|u_{i}\right|\right)-\pi / 2\right|=\arctan \left(\left|u_{i}\right| /\left|v_{i}\right|\right)$.

Corollary 2. If $\alpha=1$ then the system (1) is asymptotically stable if and only if for all eigenvalues $\lambda_{i}=u_{i}+j v_{i}$ $(i=1, \ldots, n)$ of the matrix $A$ the following conditions holds: $u_{i}<0$ and $h\left|\lambda_{i}\right|<\arctan \left(\left|u_{i}\right| /\left|v_{i}\right|\right)$.

Corollary 3. If the system (1) with $\alpha=1$ is asymptotically stable for $h=0$, then this system is asymptotically stable for all $h \in\left[0, h_{0}\right), h_{0}=\min _{i}\left\{h_{i}\right\}$, where

$$
h_{i}=\frac{1}{\left|\lambda_{i}\right|} \arctan \frac{\left|u_{i}\right|}{\left|v_{i}\right|}, \quad i=1,2, \ldots, n .
$$

The above results have been obtained in [20].

\section{Illustrative example}

Consider the fractional system (1) with $h=1$ and the matrices

$$
A=\left[\begin{array}{ccc}
0 & 1 & 0 \\
0 & 0 & 1 \\
-1 & -2 & -2.3
\end{array}\right], \quad B=\left[\begin{array}{l}
0 \\
0 \\
1
\end{array}\right]
$$

The matrix $A$ has the following eigenvalues: $\lambda_{1,2}=$ $-0.4629 \pm j 0.7165$ and $\lambda_{3}=-1.3741$.

Eigenvalues of $A$ (denoted by ' $\mathrm{o}$ ') and the stability regions $S(\alpha)$ for a few values of $\alpha$ are shown in Fig. 5. From this figure and Theorem 1 it follows that the system is stable for $\alpha=$ 0.4 and $\alpha=0.8$, and it is unstable for $\alpha=0.3$ and $\alpha=0.9$. Moreover, from Fig. 3 we conclude that for $\alpha \in[0.4,0.8]$ the system is stable and it is unstable for $\alpha \in[0.9,2)$.

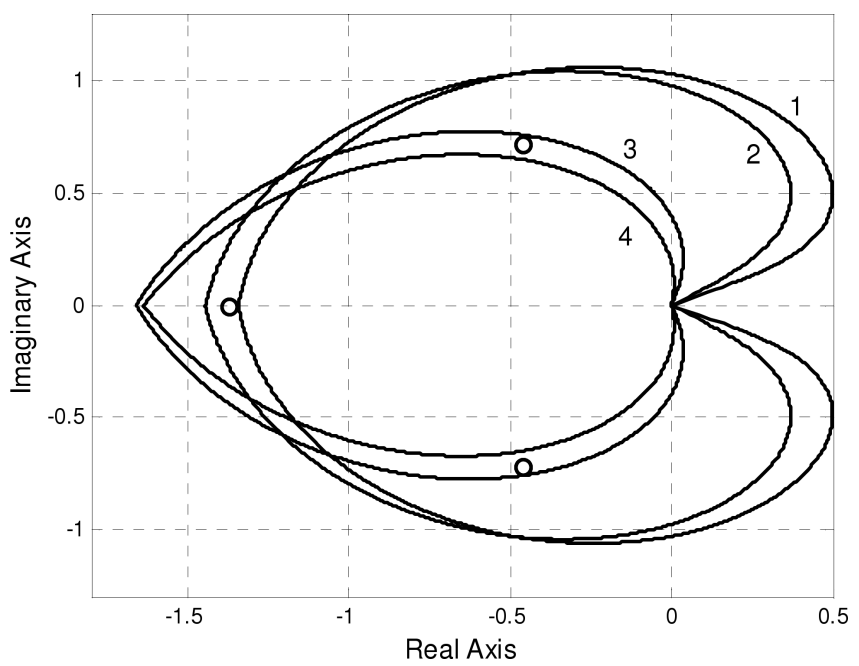

Fig. 5. Eigenvalues of $A$ ('o') and stability regions for a few values of $\alpha$ : $\alpha=0.3$ (boundary 1); $\alpha=0.4$ (boundary 2); $\alpha=0.8$ (boundary 3) and $\alpha=0.9$ (boundary 4)

If, for example, $\alpha=0.8$ then one has $\left|\arg \lambda_{1,2}\right|=2.1444$ and $\left|\arg \lambda_{3}\right|=\pi$. This means that $\left|\arg \lambda_{i}\right|>\alpha \pi / 2$ and the system with $\alpha=0.8$ is stable for $h=0$.
From Corollary 1 one has $h_{1,2}=1.0828, h_{3}=1.2670$ and $h_{0}=\min \left\{h_{1}, h_{2}, h_{3}\right\}=1.0828$. This means that if $\alpha=0.8$ then the fractional system (1), (20) is stable if and only if $h \in[0,1.0828)$.

Figure 6 shows step responses $y(t)=x_{1}(t)$ of the system for $\alpha=0.8$ and a few values of delay. The plots confirm the above result that the system is stable for $h<h_{0}$ and unstable for $h>h_{0}$.

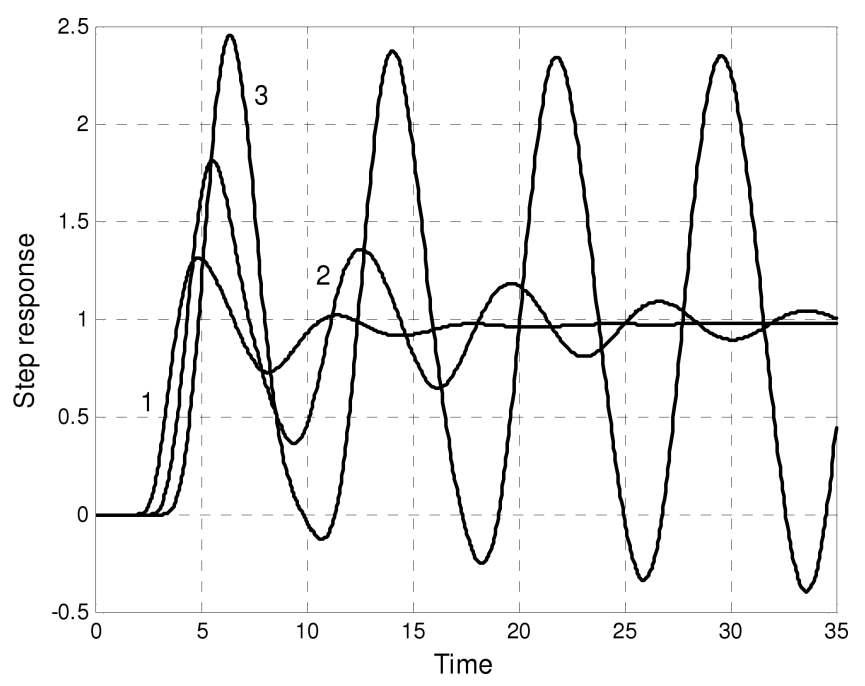

Fig. 6. Step responses for $\alpha=0.8$ and few values of delay: $h=0.7$ (plot 1$) ; h=0.9($ plot 2$) ; h=1.1($ plot 3$)$

\section{Concluding remarks}

The stability problem for continuous-time linear fractional order systems with state delay (1) has been considered. It has been shown that the system is stable if and only if all eigenvalues of the state matrix multiplied by delay in power equal to a fractional order lie in the stability region in the complex plane (Theorem 1). The new simple analytic condition for the stability are given in Theorem 2. Moreover, a new simple analytic method for computation of values of delay for which the system is stable, is derived in Corollary 1.

Acknowledgements. The work was supported by the Ministry of Science and High Education of Poland under the grant no. S/WE/1/2011.

\section{REFERENCES}

[1] S. Das, Functional Fractional Calculus for System Identification and Controls, Springer, Berlin, 2008.

[2] T. Kaczorek, Selected Problems of Fractional Systems Theory, Springer, Berlin, 2011.

[3] Y. Luo and Y-Q. Chen, Fractional Order Motion Controls, John Wiley \& Sons Ltd, Chichester, 2013.

[4] C.A. Monje, Y.Q. Chen, B.M. Vinagre, D. Xue, and V. FeliuBatlle, Fractional-order Systems and Controls Fundamentals and Applications, Springer, London, 2010.

[5] D. Matignon, "Stability result on fractional differential equations with applications to control processing", IMACS-SMC Proc. 1, 963-968 (1996). 
[6] M.S. Tavazoei and M. Haeri, "Note on the stability of fractional order systems", Mathematics and Computers in Simulation 79, 1566-1576 (2009).

[7] M. Busłowicz, "Stability of state-space models of linear continuous-time fractional order systems", Acta Mechanica et Automatica 5, 15-22 (2011).

[8] R. Stanisławski and K.J. Latawiec, "Stability analysis for discrete-time fractional-order LTI state-space systems. Part I: New necessary and sufficient conditions for asymptotic stability", Bull. Pol. Ac.: Tech. 61 (2), 353-361 (2013).

[9] R. Stanisławski and K.J. Latawiec, "Stability analysis for discrete-time fractional-order LTI state-space systems. Part II: New stability criterion for FD-based systems", Bull. Pol. Ac.: Tech. 61 (2), 363-370 (2013).

[10] M. Busłowicz and A. Ruszewski, "Necessary and sufficient conditions for stability of fractional discrete-time linear state-space systems", Bull. Pol. Ac.: Tech. 61 (4), 353-361 (2013).

[11] T. Kaczorek, "Practical stability of positive fractional discretetime systems", Bull. Pol. Ac.: Tech. 56 (4), 313-317 (2008).

[12] T. Kaczorek, "New stability tests of positive standard and fractional linear systems", Circuits and Systems 2, 261-268 (2011).

[13] M. Busłowicz, "Stability of linear continuous-time fractional order systems with delays of the retarded type", Bull. Pol. Ac.: Tech. 56, 319-324 (2008).

[14] M. Shi and Z. Wang, "An effective analytical criterion for stability testing of fractional-delay systems", Automatica 47, 2001-2005 (2011).

[15] A.R. Fioravanti, C. Bonnet, H. Özbay, and S. Niculescu, "A numerical method for stability windows and unstable root-locus calculation for linear fractional time-delay systems", Automatica 48, 2824-2830 (2012).

[16] A. Mesbahi and M. Haeri, "Stability of linear time invariant fractional delay systems of retarded type in the space of delay parameters", Automatica 49, 1287-1294 (2013).

[17] E. Kaslik and S. Sivasundaram, "Analytical and numerical methods for the stability analysis of linear fractional delay differential equations", J. Computational and Applied Mathematics 236, 4027-4041 (2012).

[18] H. Li, S.M. Zhong, and H.B. Li, "Stability analysis of fractional order systems with time delay", Int. J. Mathematical, Computational Science and Engineering 8 (4), 400-403 (2014).

[19] H. Zhang, D. Wu, J. Cao, and H. Zhang, "Stability analysis for fractional-order linear singular delay differential systems", Hindawi Publishing Corporation, Discrete Dynamics in Nature and Society ID 850279, http://dx.doi.org/10.1155/2014/850279 (2014).

[20] M. Busłowicz, "Simple stability criterion for a class of delay differential system", Int. J. Systems Science 18 (5), 993-995 (1987).

[21] M. Barszcz and A.W. Olbrot, "Stability criterion for a linear differential-difference system", IEEE Trans. Autom. Control AC-24 (2), 368-369 (1979).

[22] T. Mori and E. Noldus, "Stability criteria for linear differential difference systems", Int. J. Systems Science 15 (1), 87-94 (1984).

[23] E.N. Gryazina, B.T. Polyak, and A.A. Tremba, " $D$ decomposition technique state-of-the-art", Automation and Remote Control 69 (12), 1991-2026 (2008).

[24] T. Kaczorek, Theory of Control, PWN, Warsaw, 1977 (in Polish). 